\title{
VERTENTES TEÓRICAS E METODOLÓGICAS DA GESTÃO DE RESÍDUOS SÓLIDOS URBANOS
}

\author{
H.Domingos ${ }^{1 *}$; J.R.Pires Manso' ${ }^{1}$; A.M.M.Faria ${ }^{2}$ \\ 1 Universidade da Beira Interior/NECE, Covilhã, Portugal \\ 2 Universidade Federal de Mato Grosso/Faculdade de Economia, Cuiabá, Brasil \\ *helde@ubi.pt
}

Artigo submetido em 24/09/2015 e aceito em 30/12/2015

\begin{abstract}
RESUMO
Este artigo possui três objetivos: i) realizar a revisão de literatura atualizada do setor de resíduos sólidos urbanos (RSU), i.é, reunir o material bibliográfico em termos de pesquisa científica recente; ii) identificar as principais orientações de estudos de fundo empírico e teórico, seguidas pela investigação moderna; iii) hierarquizá-las considerando escopo econômico, política pública, ambiental e gestão do sistema operador. Este trabalho foi realizado por meio da revisão de estudos do setor de resíduos de conteúdo publicado nas principais revistas internacionais. Os resultados parecem sugerir não haver
\end{abstract}

um padrão único na abordagem destes estudos, mas, contém elementos-chave sob o guarda-chuva teórico "custo" e "eficiência". A escassez de literatura sobre a estrutura produtiva do setor de reciclagem é central no debate atual sobre RSU para convergência de abordagens sobre custos contábeis e econômicos, custo de transação, logística, externalidades e falhas de mercado. Esta indagação é fundamental para lançar luzes na direção e no escopo das pesquisas sobre RSU em debates interdisciplinares tanto acadêmicos quanto políticos.

PALAVRAS-CHAVE: resíduos sólidos urbanos, gestão ambiental, políticas públicas

\section{THEORETICAL AND METHODOLOGICAL ASPECTS OF SOLID WASTE MANAGEMENT}

\begin{abstract}
This article has three aims: i) to carry out a current literature review of solid waste sector (MSW); ii) identify the main directions of studies of empirical and theoretical background, followed by modern research; iii) hierarchically them considering the economic scope, public policy, environmental management and operator system. This study was conducted by reviewing studies on the waste sector with content published in major international journals. The results seem to suggest there is no single standard approach in these studies, but contains key elements under the theoretical umbrella
\end{abstract}

"cost" and "efficiency." The lack of literature on the production structure of the recycling sector is central issue in the current debate on MSW for convergence of approaches on account and economic costs, transaction costs, logistics, externalities and market failures. These questions is crucial to shed light to ways and scope of research on MSW at interdisciplinary and political discussions.

KEYWORDS: municipal solid waste, environmental management, public policies 


\section{INTRODUÇÃO}

A mitigação de externalidades negativas, em especial as geradas por resíduos sólidos urbanos (RSU) tem estado no centro das atenções em vários países no mundo. A discussão tem ocorrido em quatro níveis geopolíticos: local, regional, nacional e nos últimos anos transnacional (Antonioli e Massarutto, 2012). Os impactos ambientais da produção e as políticas públicas de mitigação de RSU são questões cada vez mais proeminentes na agenda das nações, pois, geram elevados custos econômicos decorrentes da sua eliminação (Mazzanti e Zoboli, 2008). Nos países em via de desenvolvimento (PVD), o modelo dominante de mitigação deste tipo de externalidade tem sido o recurso ao aterramento sanitário (Read et al., 1997). Nos países desenvolvidos (PD) a aposta tem sido na reciclagem dos resíduos inorgânicos e compostagem dos orgânicos ou incineração de ambos, enviando o restante para o aterro controlado. Contudo, a dependência do aterro sanitário ainda é relativamente alta em todo o mundo (Mazzanti e Zoboli, 2008).

Países como o Japão, os Estados Unidos da América (EUA) e muitos Estados-membros da União Europeia (UE) têm pautado estratégias de mitigação nas políticas públicas conjugadas com a recuperação de matéria e energia (European Commission, 2006), refletindo, assim, o "custo de oportunidade" como fonte geradora de benefícios, tanto econômico quanto socioambiental. A ênfase dada na recuperação material e energética desloca a discussão da fronteira de produção deste setor para além da formação dos tradicionais monopólios legais que ocorrem nos serviços quanto ao seu gerenciamento (Antonioli e Massaruto, 2012; Masui, et al., 2000; Cruz et al., 2014).

As contribuições da literatura sobre RSU enfatizam que a raiz do problema está ligada à capacidade limitada dos aterros sanitários e o volume de geração que tem crescido exponencialmente à escala global. Este entendimento foi causa determinante da "revolução silenciosa" que tem vindo a ser refletido nas legislações na Europa, Japão e EUA em passado recente, na sequência dos alertas às autoridades governamentais para melhorarem o planejamento das estratégias de mitigação, em busca de novas perspectivas de bem-estar social, econômico e ambiental (Hansen et al., 2002; Okuda e Thomson, 2007; Cruz et al., 2014). No campo regulatório, a Diretiva 94/62/CE da UE, estabelece um marco nos sistemas de gerenciamento, por responsabilizar pelo problema os agentes econômicos envolvidos na geração, distribuição, importação e utilização de produtos embalados (Bailey, 1999). Em termos de política pública a UE tem incentivado cada vez mais a via dos três " $R$ " - reduzir, reutilizar e reciclar, impondo controle mais rigoroso das ameaças que a exposição inadequada representa para a economia do setor público e aos danos ambientais, cujos reflexos recaem sobre a sociedade, além do empobrecimento dos recursos naturais, exigindo uma ação mais firme de proteção aos níveis nacionais de cada Estado e, em especial, ao nível global da UE (European Commission, 1999).

No entanto, subsistem diferenças substanciais ao nível de produção de resíduos de país para país, diferenças que afetam significativamente a forma como cada Estado lida com o problema; em grande parte essas diferenças são devidas às diferentes estruturas industriais e socioeconômicas dos países (EEA, 2010). Países desenvolvidos como o Japão, a Suécia, a Bélgica e a Dinamarca, reaproveitam acima de $90 \%$ de seus resíduos. Situações inversas podem ser observadas em alguns países da Ásia e da América Latina estando geralmente associadas aos problemas estruturais do sistema básico de recolhimento, pois, nem todos os resíduos são coletados não podendo, por isso, receber o adequado tratamento. O cenário agrava-se quando a análise incide sobre regiões com levada densidade demográfica como a China, a Índia, o México e o Brasil, países em que cerca de $90 \%$ dos resíduos sólidos produzidos nestes espaços não recebem o tratamento em locais de deposição, transformando-se em uma poderosa fonte de catalisação e de emissão de dióxido de carbono $\left(\mathrm{CO}_{2}\right)$ e gás metano $\left(\mathrm{CH}_{4}\right)$ para atmosfera (Lino e Ismail, 2011). 
Entre a variedade de serviços contratados pelo setor público inclui-se o gerenciamento municipal dos resíduos. Embora a contratação dos serviços de coleta seja simples, por exemplo, para atividades de ação mitigadora, a implantação de um sistema de recuperação de materiais é muito complexa. Neste sentido, encontrar formas de fornecer um serviço de gestão com qualidade e baixo custo para o setor público conjugado com a reciclagem é o principal desafio para fomentar a redução de RSU nos centros urbanos (Walls, 2005). Desta forma, o gerenciamento de resíduos sólidos nessas áreas para ser sustentável depende da participação do Estado, do setor econômico e da sociedade (Ezebilo e Animasau, 2011).

Analisar o abrangente cenário com múltiplos fatores envolvendo vantagens econômicas e ambientais do setor de resíduos sólidos é extremamente complexo, pois, envolvem diferentes campos de análise teórica e metodológica. Este trabalho tem como objetivo fornecer uma revisão de estudos sobre RSU, partindo-se de um escopo abrangente em direção a uma estrutura mais organizada e mais prática, identificando as principais vertentes de estudos teóricos e empíricos. A motivação de estudar este assunto surge da necessidade de realizar a montagem do quebra-cabeça que é a literatura multivariada do tema RSU, buscando hieraquizar os diferentes estudos considerando o seu enquadramento econômico, de política pública, de meio ambiente e da gestão do sistema operador. Em geral os estudos sobre RSU analisam modelos de gerenciamentos enfatizando aspectos técnico-institucionais, quase sempre com o objetivo de minimizar os custos de operação do sistema (Walls et al., 2005; Bel e Miralles, 2003), mas pouco em identificar potenciais de agregação de valor e utilização de materiais recuperáveis.

Desta forma, a literatura tem mostrado que esses estudos ignoram em suas análises importantes fatores econômicos no lado da reciclagem, como o impacto da produção nas economias locais, a agregação de valor da produção, o transbordamento financeiro gerado pela cadeia produtiva e arregimentação de força de trabalho. As inclusões destes fatores na investigação aumentam a necessidade de uma visão mais ampliada da literatura sobre o setor de resíduos sólidos. Neste contexto, a principal contribuição deste artigo é lançar luz na importância que as condições estruturais de "produção", de "economia" e de "política pública" têm quanto à prevenção de danos e impactos gerais evitados pela mitigação no nível da reciclagem, cujo efeito do uso da estrutura produtiva pode exercer sobre importantes fontes de sintropia, designadamente o petroléo, a bauxita, os minérios de ferro e a base florestal.

O artigo para além desta introdução tem uma segunda seção que analisa o enquadramento teórico e metodológico do setor de RSU; uma terceira que a apresenta a abordagem das principais modelagens encontradas na literatura; uma quarta que apresenta uma abordagem das políticas públicas do setor de resíduos sólidos, e por fim, uma quinta, a conclusão com uma síntese do que mais importante se deixou ao longo de toda a investigação sobre o mercado de resíduos sólidos.

\section{MATERIAIS E MÉTODOS}

Foi realizada uma busca na base de dados Scopus e ISI com os termos "municipal solid waste", "waste management", "value of solid waste" and "waste". Os resultados indicaram uma variedade de literaturas internacionais e foram selecionadas 78 que demonstraram elevado impacto de citação.

A visão sistêmica da literatura sobre resíduos sólidos é direcionada para as estruturas teóricas e metodológicas de mitigação baseadas nas políticas de comando e controle. A partir do ano 2000 essas políticas passaram a sofrer algumas alterações com a introdução de novas abordagens por meio de instrumentos de comunicação social, no sentido de despertar os valores sociais, como por exemplo mensagens do tipo "não jogue lixo na rua", "separe lixo seco de úmido", 
e outras ações cognitivas. No campo dos instrumentos econômicos surgem políticas de impostos, subsídios diretos, valores creditícios fiscais, licenças para emissão em sistemas de negociação e/ou direitos adquiridos (Fischer, 2008; Lehman, 2012; Grunningham e Grabosky, 1998).

Shmelev e Powell (2006) argumentam que a década de 1990 ficou marcada pelas diversas tentativas de aprimorar os estudos no sentido de analisar a eficiência do sistema de monitoramento e gestão de RSU. Para tanto, as análises foram realizadas considerando diferentes fatores econômicos e socioambientais. Estes mesmos autores referem que as pesquisas não conseguiram demonstrar uma imagem holística dos sistemas de gestão, onde os fatores identificassem impactos de relevâncias, bem como a interferência de outros processos como tecnologia, logística, e principalmente as partes afetadas pela cadeia de distribuição do setor de RSU. Para Marques et al., (2012) a literatura disponível sobre este setor econômico apresenta-se escassa. Contudo, a literatura elencada com abordagem dos problemas ambientais e dos fatores econômicos assenta no desempenho de políticas simples ou na melhor das hipóteses na comparação de duas ou mais políticas de comando e controle (Lehmann, 2012).

A revisão desta literatura permitiu identificar cinco vertentes de investigações de fundo empírico e teórico que podemos hierarquizar da seguinte maneira: i) geração e tratamento de resíduos que envolvem custos, nomeadamente economias de escala e de escopo; ii) tecnologia e gestão de aterros, abordados pelo prisma da eficiência e custo; iii) sistema operador da gestão, público e privado no sentido de propriedade; iv) abordagem macroeconômica do mercado de resíduos, uma interpretação do setor através da análise de fileira dos resíduos com uso de matriz insumo-produto; e por fim, v) análise da relação causal entre renda per capita e emissão de resíduos, sob a hipótese da Curva de Kuznets Ambiental (Read et al., 1997; Nakamura, 1999; Masui et al., 2000; Ferrer e Ayres, 2000; Jaffe et al., 2005; Shmelev e Powell, 2006; Lavee, 2007; Okuda e Thomson, 2007; Simões et al., 2010; Bae, 2010; Simões e Marques, 2012; Antonioli e Massaruto, 2012, Cole at al.,1997; Yanrong et al., 2011).

\section{RESULTADOS E DISCUSSÃO}

Essa seção reúne as cinco vertentes de abordagem identificadas. O objetivo não é esgotar cada tema, mas apresentar as suas principais indagações e resultados, bem como oferecer à comunidade acadêmica e aos gestores de RSU quais as principais literaturas disponíveis a serem consultadas. Ademais, pode servir de referência e guia de consulta na avaliação de projetos que envolvam a gestão de RSU.

\subsection{Geração e tratamento de resíduos}

Os primeiros passos para compreender a geração e o tratamento de RSU é proceder à sua caracterização, abrangendo todo o sistema integrado de gestão em operação que tem pautado cada vez mais na melhoria da eficiencia no tratamento de RSU (Metin et al., 2003). Garechana et al., (2014) notam existir uma extensa e complexa cadeia produtiva a montante, que não pode ser ignorada, que envolve interações de atividades de reorganização, de classificação e de separação de materiais, desempenhadas principalmente pelo setor de serviços. Após esses diferentes processos produtivos, a jusante se transformam os RSU em novas matérias-primas pelo setor industrial, fechando um ciclo de throughput.

Massarutto (2007) define essa amplitude de mercado em três fases inter-relacionadas: a primeira delas diz respeito ao fornecimento de serviços necessários para a atividade de 
recolhimento; a segunda, o mercado de manuseamento e eliminação, e a terceira à reciclagem. Outros aspectos não menos importantes referem-se à estrutura de mercado quando a matéria-prima reciclada é reincorporada no ciclo econômico pelo segundo setor, requerendo novas interpretações de base teórica e econômica.

Estudos abordando estes assuntos remetem a pesquisas realizadas por Smith (1972), cujas análises são aplicadas à realidade dos EUA e tinham como objetivo contrastar os percentuais ótimos de reciclagem e a deposição final nos aterros sanitários. Lavee (2007) e Masui et al., (2000) identificam o custo direto de recolhimento, deposição e incineração em relação à reciclagem. Nessa vertente de pesquisa o custo é o indicador mais usado, denotando-se, os custos de recolhimento menores que os custos de reciclagem, e a presença de elementos que favoreciam a alternativa da deposição final com melhores condições do que a reciclagem nos processos de gestão. Estes resultados vão de encontro a Massaruto et al., (2011) que confirmaram existirem "custos extras" aos operadores dos sistemas de gestão privado e público no segundo e terceiro setor, indústria e serviços, respectivamente, quando estes empregam a reciclagem como instrumento de mitigação deste tipo de externalidades.

Todavia, em dois estudos anteriores, Lavee (2007) e Nakamura (1999) argumentam que mesmo ignorando custos externos de gerenciamento de aterros sanitários empregando a reciclagem, os municípios poderiam ser eficientes. Esta dicotomia é respaldada pela dinâmica do sistema de gestão, pois, neste caso, não somente os custos de recolha e tratamento atual, mas também os custos futuros com os aterros sanitários deveriam ser considerados. Além disso, a recuperação material e energética para estes autores conforma a principal alternativa de mitigação do problema da falta de espaço nos aterros e da escassez de locais para implantação de novas unidades sanitárias.

Quanto à estrutura de mercado, um estudo realizado por Callan e Thomas (2001), demostrou que o setor de resíduos sólidos apresentava economia de escala de produtos e economia de escopo no mercado como um todo. Estes autores acreditavam existir uma complementariedade de custos entre reciclagem e deposição final e concluíram existirem retornos crescentes de escalas para reciclagem e retornos constantes de escala considerando a deposição final e/ou incineração, respectivamente. No entanto, Bohm et al., (2010: 870), estimando funções de custos demonstraram haver economias de escalas em ambas as atividades, mas que desapareciam à medida que a participação da reciclagem era aumentada. Outros estudos fornecem extensas avaliações sobre economias de escalas no setor de RSU (ver Lavee e Khatib, 2010; Abrate et al., 2011; Carroll, 1995; Folz, 1999; Weng e Fugiwara, 2011; Bel e Fageda, 2010).

\subsection{Tecnologia e gestão de aterros sanitários}

A segunda vertente prende-se com a utilização de tecnologias e com o formato de gerenciamento empregado pelos gestores de aterros sanitários. A organização deste setor e as questões tecnológicas podem ser avaliadas por intermédio do desempenho de eficiência e da capacidade de inovação produtiva (Peroto et al., 2008). Todavia, o método empregado é dependente do tipo de política adotada por cada localidade, conforme Massui et al., (2000) afirmam que a população no Japão considera o tipo de política pública para RSU muito importante devido a elevada densidade demográfica em relação ao reduzido espaço; inclusive suas zonas rurais estavam recebendo parte de RSU gerados no eixo mais dinâmico da economia japonesa; esta condição, dentre outras variáveis demonstraram a sobrecarga da capacidade de gestão de RSU do país.

Okuda e Thomson (2007) confirmaram que a gestão de RSU no Japão passou por uma fase crítica devido à escassez de espaço territorial e por encontrar forte resistência da população baseada no NIMBY (Not In My Back Yard), o que se refletiu em mudanças significativas das legislações 
sobre o planejamento do uso do solo, este tipo de preocupação foi iniciado na Inglaterra e ganhou adeptos em diversas outras partes do mundo. Estudos sobre este tipo de abordagem dos RSU são os de Gandy (1994) e Adams (1996). Ainda segundo Okuda e Thomson (2007) este tipo de apelo popular teve reflexo direto nas decisões de políticas públicas voltadas a resolver o problema de gestão de RSU no Japão, cuja capacidade de integração dos municípios converteu-se em solução ótima levando o país a realizar a mudança na forma de eliminação tradicional de RSU, por trocar incineradores de custos elevados e altamente poluentes para tecnologias de reciclagem. O resultado desta mudança colocou o Japão na vanguarda da indústria de transformação de RSU, país que se tornou líder mundial na recuperação de matéria-prima a partir de RSU.

Em geral o sistema de gerenciamento e de organização em relação às questões tecnológicas (incineração, recuperação de matéria, energia, e o tratamento de resíduos) são aspectos importantes relacionados diretamente com a participação do setor privado no mercado de RSU (Buclet e Godard, 2000; Bertossi et al., 1996). De acordo com Lehman (2012) este tipo de abordagem tecnológica observa a geração de um tipo de externalidade positiva que advém de "spillover tecnológico" gerado por empreendedores inovadores que contribuem para alterações tecnológicas, impactando diretamente na forma como as políticas públicas são conduzidas na resolução de problemas de resíduos no meio ambiente. Conforme afirmado por Jaffe et al., (2005), políticas de comando e controle baseadas em tecnologias podem servir como complemento de controle de poluição.

\subsection{Propriedade pública versus privada}

A terceira vertente enfatiza questões estruturais de mercado quanto à eficiência e à operacionalização dos serviços no recolhimento e tratamento de RSU nos municípios. Os estudos que analisam estas questões do ponto de vista da propriedade reconhecem a sua relevância em função do impacto econômico e do desempenho que a atividade exerce sobre a economia (Simões et al., 2010). O setor econômico de resíduos, especialmente o serviço de coleta ou recolha, possui uma participação acentuada da iniciativa privada, fato que leva à discussão público versus privado (Bae, 2010).

Antoniolli e Massaruto (2012) propuseram a identificação de possíveis implicações relacionadas com a concorrência e regulação econômica, devido o interesse privado no sistema de gerenciamento de RSU e concluíram que esta questão serviu para adicionar novos desafios no que diz respeito à fronteira entre o serviço operado pelo setor público e o operado no domínio de mercado. Atualmente, os principais fatores competitivos no negócio de resíduos sólidos são a rentabilidade para o lado do setor privado, e os custos de transação mais baixos para o controle do setor público. Ainda segundo Antonioli e Massarutto (2012) o surgimento de recuperação e reciclagem de RSU significou uma pedra angular na questão da gestão de RSU e iniciou uma nova fase de obrigações por parte do serviço público no que diz respeito ao cumprimento de metas.

Marques et al., 2012 utilizando dados de 23 empresas referentes aos anos de 2006 a 2010 para Portugal com metodologia DEA (Data Envelopment Analysis) diagnosticaram ineficiências acentuadas na gestão dessas empresas, que associaram à falta de incentivos relacionados com o baixo rendimento dos sistemas de reciclagem. Note-se, que a experiência de Portugal no campo regulador é impar, é um dos poucos países no mundo a instituir uma entidade reguladora para resíduos, o Instituto Regulador de Águas e Resíduos (IRAR), antecipando-se a frente para o enfrentamento dos novos desafios que representa o combate e/ou equilíbrio entre produção e eliminação de resíduos sólidos na perspectiva de bem-estar econômico e socioambiental. Dos 29 serviços de RSU em Portugal 17 são concessões, 14 são controladas pelo Estado e três são operados 
pela iniciativa privada (Simões et al., 2010). Ainda no âmbito da discussão público versus privado, empresas públicas dominam o mercado de recolhimento na Espanha, Áustria, Alemanha, Itália e Suécia enquanto o setor privado está presente com mais frequência na Holanda, Reino Unido, França, Bélgica e Portugal (Antonioli e Massarutto, 2012).

A literatura aponta esta tendência de acordo com o interesse de cada agente envolvido neste tipo de transação. Entendendo que a eficiência do setor de resíduos sólidos pode ser observada sob quatro aspectos, o primeiro do ponto de vista do operador do sistema, cujo objetivo é o da minimização do custo com vislumbre do lucro; o segundo diz respeito, ao interesse público da sociedade quanto a receber um serviço de qualidade, uma vez que o contribuinte é onerado pelo serviço e em contrapartida deseja atingir suas metas de desenvolvimento pautado no bem-estar; o terceiro refere-se aos interesses governamentais, que pretendem solucionar o problema ao menor custo possível; o quarto consiste na indústria de reciclagem em maximizar o fluxo de resíduos e suas quantidades como forma de garantir a sobrevivência e lucro no mercado. Essa aglutinação de fatores e interesses consistem em um único objetivo do ponto de vista econômico, que é a escolha do tipo de prestação de serviço de RSU que se espera para ambos os lados, oferta e demanda dos serviços de resíduos sólidos (Shmelev e Powell, 2006; Walls et al., 2005; Bel e Miralles, 2003).

Neste modelo dual há muitos tipos de arranjos envolvendo ambas as participações, assim servindo os diferentes interesses. A mais controversa é a privatização dos serviços públicos de gerenciamento de RSU, pois têm aumentado gradualmente tanto pela sua importância econômica que tem atingido altos volumes monetários como pelos milhares de empregos criados (Antonioli e Massaruto, 2012). A privatização pode ser entendida como a transferência de propriedade das instalações físicas do setor público para o privado. Estes tipos de serviços têm características naturais de monopólio (Demsetz, 1968), mas, por outros tipos de concessões o serviço pode ser entregue também a iniciativa privada por terceirização (Bel e Miralles, 2003). Neste caso, este tipo de negócio gera ganhos financeiros aos contratantes que fazem subcontratações, e isto demonstra a importância fundamental do lucro para a propriedade privada (Vickers e Yarrow, 1991).

Sobre os ganhos de eficiência do modelo público versus privado, Bae (2010) concluiu que o serviço público de coleta de RSU é mais eficiente do que o serviço prestado pela iniciativa privada. No entanto, este autor, que não considerou os efeitos sobre a qualidade destes serviços, apontou que não houve diferenças nos custos dos serviços decorrentes da operação por empresa pública ou privada. Embora, o setor público possa ter maiores ganhos de eficiência e a liderança de mercado, a maior parte do valor adicionado da indústria acontece no lado das empresas que operam o sistema de recolha conjugado com a reciclagem (Antonioli e Massarutto, 2012).

\subsection{Abordagem macroeconômica do mercado de resíduos}

A quarta vertente ou linha de investigação diz respeito à visão macroeconômica do mercado de resíduos sólidos. As primeiras investigações referentes à indústria de papel dos EUA com materiais recuperados foram discutidas por Nestor (1992). Posteriormente outros autores fizeram comparações de taxas de utilização de papel reciclado no mercado. Hervani (2005) identificou o poder de oligopsônio na indústria de reciclagem de papel usando dados sobre preços de insumos entre 1972-1995 o que garantia a estrutura industrial da época uma margem maior de preço-custo no mercado. Berglund e Söderholm (2003) fizeram a comparação das diferenças de taxas de recuperação e utilização de resíduos de papel por 49 países utilizando séries temporais e crosssection em dados em painel e concluíram que a taxa de recuperação era dependente de fatores econômicos, densidade demográfica e competitividade no mercado mundial de papel. 
Nakamura (1999) usou um quadro matricial com setores afetados pela cadeia de produção e distribuição do mercado de resíduos sólidos. Nesta investigação o mercado de resíduos sólidos é estudado recorrendo a uma estrutura macroeconômica. Na mesma linha de investigação Massui et al., (2000) e Ferrer e Ayres (2000) avaliaram as atividades econômicas convencionais e ambientais, além do impacto da recuperação desse material de resíduos no seu retorno ao ciclo econômico como matéria-prima. Ambos os estudos utilizaram matrizes insumo-produto e os resultados sugeriram que aumentando os índices de reciclagem se obtêm impactos positivos no progresso econômico devido à melhoria das condições ambientais, mas também pela formação de poupança da matéria-prima virgem devido à exploração evitada pelos RSU recuperados que retorna, assim, ao sistema econômico, em um sistema de throughput.

Além disso, estes efeitos afetam positivamente os agregados macroeconômicos pela promoção de demanda por trabalho, na redução do nível de transação interindustrial e da geração de contribuições significativas ao nível da sua estrutura industrial contribuindo com a redução da emissão de dióxido de carbono $\left(\mathrm{CO}_{2}\right)$ e gás metano $\left(\mathrm{CH}_{4}\right)$, consequência do desvio de material dos aterros sanitários para a reciclagem, favorecendo assim a economia das fontes de sintropia, com a utilização de forma sustentável dos resíduos por meio da reciclagem (Nakamura, 1999; Ferrer e Ayres, 2000).

\subsection{Renda per capita e emissão de resíduos sólidos}

$\mathrm{Na}$ quinta e última vertente de investigação sobre resíduos sólidos, alguns autores buscam evidência empírica de tendências do aumento da produção de RSU em função do nível de renda per capita e/ou do desenvolvimento dos países. Bringezu et al., (2003) afirmam que este ramo de pesquisa (nos RSU) é menos desenvolvido do que avaliações sobre outros tipos de poluição, como efluentes lançados no ar ou nas bacias hídricas. Além disso, os dados de grupos de países são heterogêneos, o que implica problemas na análise, pois se os dados forem homogêneos poderiam fornecer uma estrutura de dados mais confiável para avaliação de políticas públicas mais sólidas (Mazzanti e Zoboli, 2008). Muitos autores têm buscado respostas centrando-se sobre a Curva de Kuznets Ambiental (CKA), que descreve a relação de U invertido entre diferentes poluentes e a renda per capita. Dessa forma, acredita-se que os níveis de renda podem afetar significativamente os cuidados com a ação antrópica sobre o meio ambiente, encorajando a sociedade a cobrar de seus representantes políticas públicas de reciclagem de resíduos sólidos (Chen e Chen, 2008). A lógica deste processo é que quando se eleva a renda per capita acima de determinado patamar, a poluição naquela sociedade começa a reduzir.

A partir dessas preocupações sugiram pesquisas focadas no impacto que a emissão de resíduos sólidos exerce sobre a sociedade em termos econômicos e ambientais. O primeiro estudo testando a CKA com dados de resíduos sólidos foi realizado por Cole et al., (1997). Seus resultados não identificaram evidência de $U$ invertido na relação entre geração de RSU crescente e renda. Outros estudos testaram a hipótese CKA para resíduos sólidos e a renda, contudo, os resultados não são unânimes (ver Leigh, 2004, Seppälä et al., 2001; Berrens et al., 1998; Wang et al., 1998; Fischer Kowaslki e Amann, 2001, Mazzanti e Zoboli, 2005; Yanrong et al., 201; Chen e Chen, 2008).

Conforme afirmado por Mazzanti e Zoboli (2008), a literatura demonstra falta de investigação aprofundada dessas forças motrizes, uma vez que os níveis de reciclagem e compostagem estão positivamente relacionada como o crescimento econômico, o que confimaria a hipótese da CKA. Desta forma, ainda há muitas dúvidas na associação de evidências que sinalizam os efeitos de diferentes estruturas sociais, econômicas, tecnológicas e políticas na prevenção de resíduos e/ou sua mitigação. São necessárias novas investigações com metodologias apropriadas 
para entender porque em alguns espaços a hipótese da CKA se ajusta para RSU e em outros ainda não ocorre.

\subsection{A modelagem aplicada em estudos sobre resíduos sólidos}

Os estudos empíricos apreciados utilizam diversos indicadores de poluição que causam degradação ambiental para avaliar qualidade do ar, da água, dos RSU e do desflorestamento (Dinda, 2004). Em termos metodológicos utilizam técnicas econométricas para avaliar o desempenho das atividades econômicas, tanto na prestação de serviço de recolha quanto na reciclagem. Custo e eficiência são os fatores mais estudados com recurso a modelos paramétricos e não paramétricos que ajudam a compreender as estruturas de mercado e o nível de eficiência alcançado pelas empresas. Informações quantitativas e qualitativas referentes ao volume, peso, abrangência da área, e frequência de coleta são os principais indicadores capazes de afetar significativamente o custo dos serviços (ver Pier et al., 1974; Stevens, 1978; Tickner e McDavid, 1986; Szymanski e Wilkins, 1993; Callan e Thomas, 2001; Lavee, 2007; Bohm et al., 2010; Simões e Marques, 2012).

Dentre as metodologias que fornecem ao gestor de RSU alternativas de escolha de gerenciamentos o modelo mais usado é o da avaliação de ciclo de vida, ferramenta que consegue reunir diferentes fatores e avaliar uma série de possíveis impactos associados ao fornecimento do serviço prestado apontando uma determinada padronização dos resultados (Cruz et al., 2012; Barton et al., 1996; Highfill e McAsey, 1997). Outros trabalhos abordam problemas semelhantes enfrentados por administradores de aterros sanitários, serviços de recolha e do mercado de reciclagem. Dentre a variedade de ferramentas econométricas, destacam-se, a análise multicritério; a análise de regressão múltipla; as técnicas de avaliação de impacto ambiental (EIA) bastante criticadas por incluírem altos graus de subjetividade e por último, técnicas que recorrem à matriz insumo-produto para avaliar o impacto econômico nos diversos setores da cadeia de RSU a partir de reinserção de matéria-prima reciclada (Shmleve e Powel, 2006; Cruz et al., 2012; Nakamura, 1999; Ferrer e Ayres, 2000; Masui et al., 2000).

As técnicas diferem de acordo com os objetivos a alcançar e essa diversidade de técnicas tem sido associada a conclusões divergentes em situações idênticas (Haas et al., 2003), que comprometem a generalização dos resultados. Porém, a interação da economia e o meio ambiente no mercado de resíduos sólidos possuem aspectos relevantes do ponto de vista endógeno e exógeno, em particular os impactos sociais desiguais, barreiras impostas por regulamentação governamental e a presença de informação assimétrica no setor. Essas falhas de mercado impõem dificuldades no desenvolvimento de aplicações capazes de integrar com maior precisão a análise do mercado (Shemlev e Powel, 2006).

\section{AS POLÍTICAS PÚBLICAS DE MITIGAÇÃO DA PRODUÇÃo DE RESÍDUOS}

Há imensa literatura econômica que investiga o uso de várias políticas - o mix de políticas para lidar com os problemas de poluição individuais. Essas políticas públicas na verdade podem exercer uma multiplicidade de metas ambientais bem como objetivos não ambientais pautados pelo desenvolvimento econômico (Lehman, 2012). A produção de resíduos continua a aumentar proporcionalmente à renda e junto os custos econômicos e ambientais associados à sua eliminação. A redução da geração de resíduos na fonte parece indicar uma alternativa válida a usar no combate à emissão de RSU. Mas, a sua redução precisa de ser estimulada, por exemplo, através da adoção de metas estabelecidas nas políticas públicas voltadas para geração per capita (Mazzanti e Zoboli, 2008), tendo eventualmente como ponto de partida as unidades familiares e o compartilhamento de 
responsabilidade na produção e dispersão de RSU como forma de lidar com o problema no longo prazo (ver Collins et al., 2006; Jenkins et al., 2003; Peretz et al., 2005).

Quando o escopo analisado é o da deposição em aterro, com ambos os efeitos ambientais e ênfase política, a análise econômica focaliza-se na avaliação custo-benefício das externalidades (Pearce, 2004). De acordo com Chen e Chen (2008) a taxa per capita de recolha de RSU das unidades familiares está intimamente associada com a sua renda. Isto revela que as políticas públicas de gestão têm que ser capazes de relacionar as características de comportamento e os valores culturais da população associados à reutilização e/ou reciclagem de RSU. Assim, a produção de benefícios ambientais e econômicos pode afetar significativamente a redução da poluição (Chen e Chen, 2008). Mazzanti et al., (2008a) usando dados da Itália, argumentam que o desvio de RSU de aterros sanitários é impulsionado principalmente pelos custos ambientais e pelas oportunidades ligadas à densidade populacional e pela imposição de impostos como instrumentos econômicos às famílias com vistas a cobrir os custos integrais dos serviços de resíduos em nível regional.

Ayres et al., (1997), posicionando-se no lado da produção, argumentou que a recuperação de bens a partir de resíduos reduz o fluxo de RSU depositados em aterros, fato que possui duas implicações do ponto de vista econômico e ambiental: (1) na esfera ambiental, o efeito de conservação de recursos; e (2) na esfera econômica, o efeito da redução de resíduos depositados em aterros aumentando a sua vida útil e gerando economias com as despesas de manutenção. Ferrer e Ayres (2000) afirmam que o principal problema que tem impedido o aumento de atividades de recuperação de resíduos é a infraestrutura inadequada, problema que se resolve com a formulação de políticas públicas bem definidas para o setor de RSU com especial atenção para o setor de reciclagem. As evidências sobre possíveis benefícios econômicos, consequência da reciclagem e do aumento paralelo dos custos de eliminação está na descoberta de um gargalo ou estrangulamento, que pode ser considerado como fator que impede a exploração rentável da reciclagem em relação à deposição final (Antonioli e Massaruto, 2012).

\section{CONCLUSÃO}

Uma boa revisão de literatura possui dois importantes impactos ou alcances: um primeiro que permite ao investigador ter a noção globalizada da orientação das pesquisas recentes auxiliando-o como ferramenta para identificar objetivos, variáveis e metodologias de um amplo arcabouço, isto é, pondo-se a par do estado da arte, neste caso, no âmbito da temática dos RSU; um segundo que permite ao investigador fazer inferências a partir das teorias observadas na argumentação das multivariadas pesquisas sobre o tema investigado.

Neste sentido, algumas questões relativas ao crescimento econômico combinado com a superação da degradação ambiental têm sido listadas em uma gama de trabalhos sobre RSU, principalmente em relação aos efeitos de externalidades negativas, ou falhas de mercado que impactam diretamente no sistema econômico e ambiental, derivados do aumento exponencial da produção de resíduos e da acumulação e emissão de gases de efeito estufa (GEE) por aterros sanitários (Kaika e Zerva, 2013).

Os resultados desta revisão de literatura parecem sugerir não haver um padrão único na abordagem ao tema dos resíduos sólidos, mas, possuem elementos que conformam um conjunto de informações sob o guarda-chuva teórico "custo" e "eficiência" pautados na observação de empresas operadoras do sistema de gestão e de reciclagem de resíduos sólidos. Além disso, é observado 
estudos com abordagem sobre a relação causal entre o efeito que a renda per capita possivelmente exerce sobre a geração de resíduos sob a hipótese da Curva de Kuznets Ambiental (CKA).

A escassa abordagem sobre a estrutura econômica de setor de reciclagem dentro da literatura é uma lacuna importante. Por suposto, esta estrutura produtiva pode agregar valor, criar empregos e impostos, além de gerar externalidades positivas pela prevenção de danos ambientais devido aos impactos evitados no nível da reciclagem de RSU, mas não tem sido objeto de análises pela comunidade acadêmica. As abordagens não apresentam uma linha mestra e a combinação de políticas públicas e de padrões tecnológicos usados no fomento da redução e/ou o equilíbrio entre geração e eliminação dos resíduos sólidos deixam de fora do escopo analítico, possíveis externalidades positivas do setor. Outra hipótese é a condição das empresas do setor de reciclagem não disponibilizar dados econômicos fiáveis sobre a produção e valor agregado alcançado no setor de reciclagem, uma vez que o setor lida com elevadas transações monetárias em contratos de prestação de serviços, denotando-se um quadro estratégico dos setores público versus privado em uma clara assimetria de informação a favor dos agentes privados.

O uso de instrumentos econômicos (IE), de comando e controle (CC) e de comunicação social (CS) são visões complementares de um movimento maior que possivelmente pode explicar porque uma determinada sociedade recicla e em que nível de custos pode ser alcançando o desenvolvimento sustentável pelo setor de resíduos sólidos. Assim, a abordagem da combinação de políticas de comando e controle e comunicação social para a gestão de RSU ajustadas à sua estrutura econômica pode indicar uma possível alternativa no nível da reciclagem de resíduos sólidos. Essas questões são centrais para os debates atuais e podem ajudar na convergência de muitas outras abordagens como os custos contábeis e econômicos, custos de transação, logística e outras falhas de mercado. Este entendimento é fundamental para lançar luzes em futuras investigações que podem subsidiar debates interdisciplinares tanto acadêmicos quanto políticos.

\section{AGRADECIMENTOS}

Os autores desejam manifestar o seu público agradecimento à Fundação Capes do Ministério da Educação do Brasil, pelo financiamento desta pesquisa; e ao NECE - unidade de investigação do Departamento de Gestão e Economia financiada pelo FCT de Portugal pelo apoio prestado na elaboração deste texto.

\section{REFERÊNCIAS}

ABRATE, G., ERBETTA, F., FRAQUELLI, G., VANNONI, D. The cost of disposal and recycling: an application to Italian municipal waste solid services. Hermes: Working paper n. 2, 2011.

ADAMS, T. (), Spotlight on the looming planning crisis. The Waste Manager, 1996.

ANTONIOLI, B., MASSARUTTO, A. The municipal Waste Management Sector in Europe: shifting boundaries between public service and the market. Annals of Public and Cooperative Economics, v.83, p.505-532, 2012.

AYRES, R., FERRER, G., VAN LEYNSEELE, T. Eco-efficiency, asset recovery and remanufacturing. European Management Journal, v.15, n.5, p. 557-574, 1997.

BAE, S. Public versus Private Delivery of Municipal Solid Waste Services: The Case of North Carolina. Contemporary economic policy, v.28: p.414-428, 2010.

BAILEY, I. Flexibility, harmonization and the single market in EU Environmental Policy: The packaging waste directive. Journal of Common Market Studies, v.37, p.549-571, 1999.

BARTON, J. R., DALLEY, D., PATEL, V. S. Life cycle assessment for waste management. Waste management. 16, p. 35-50, 1996. 
BEL, G., MIRALLES. A. Factors Influencing the Privatization of Urban Solid Waste Collection in Spain. Urban Studies, 40, 1323-34, 2003.

BEL, G., FAGEDA, X. () "Empirical Analysis of Solid Management Waste Cost: Some Evidence from Galicia, Spain. Resouces, Conservation and Recycling. v.54, p. 187-193, 2010.

BERRENS, R., BOHARA, A., GAWANDE, K., WANG, P., Testing the inverted U-hypothesis for U.S. hazardous waste: an application of the generalised Gamma model. Economic Letters, v. 55, p.435-440, 1998.

BERTOSSI, P., KAUlARD, P., LOLli, A., MASSARUTTO, A. Per una nuova política industrial nel settore dell'igiene urbana in Italia. Economia Delle Fonti Di Energia e Dellámbiente, n. 3, 1996.

BERGLUND, C., SODERHOLM, P. An econometric analysis of global waste paper recovery and utilization" Environmental and Resource Economics, v.26, n.3, p. 429-456, 2003.

BOHM, R. A., FOLZ, D.H., KINNAMAN, T.C., PODOLSKY, M.J. The Costs of Municipal Waste and Recycling Programs. Resources, Conservation and Recycling, 54, p. 864-871, 2010.

BRINGEZU, S., SCHÜTZ, H., MOLL, S. Rationale for and Interpretation of Economy-Wide Materials Flow Analysis and Derived Indicators. Journal of Industrial Ecology, 7, p. 43-64. 2003.

BUCLET, N., GODARD, O. Municipal Waste Management in Europe: a Comparative Study in Building Regimes. Kluwer, Amsterdam, 2000.

CALlAN, S. J., THOMAS, J.M. Economies of Scale and scope: A Cost Analysis of Municipal Solid Waste Services. Land Economics, v.77, n4, p. 548-560, 2001

CARROLL, W. The Organization and Efficiency of Residential Recycling Services. Eastern Economic Journal, v.21, p. 215-225, 1995.

CHEN, C. C., CHEN, Y. T. Income Effect or Policy Result: a Test of the Environmental Kuznets Curve. Jounal of Cleaner Production, v.16, n.1, p. 59-65, ISSN 0959-6526, 2008.

COLE, M., RAYNER, A., BATES, J. The EKC: an empirical analysis. Environment and Development Economics, v.16, n.4, p. 401-416, 1997.

COLlins, A., O’DOHERTY, R., SNELL, M. C. Household Participation in Waste Recycling: Some National Survey Evidence from Scotland. Journal of Environmental Planning and Management, v.49, n.1, p. 121-140, 2006.

CRUZ, N. F., SIMÕES, P., MARQUES, R. C. Economic Cost Recovery In The Recycling of Packaging Waste: The Case of Portugal, Journal of Cleaner Production, v. 37, p. 8-18, ISSN 0959-6526, 2012.

CRUZ, N.F., FERREIRA, S., CABRAL, M., SIMÕES, P., MARQUES, R. C. Packaging Waste Recycling In Europe: Is The Industry Paying For It?. Waste Management, v. 34, n.2, p. 298-308, ISSN 0956-053X, 2014.

DEMSETZ, H. Why Regulate Utilities?” Journal of Law and Economics, v.9, p.55-65, 1968.

DINDA, S. Environmental Kuznets Curve hypothesis: a survey. Ecological Economics, v.49, p.431-455, 2004.

EEA. O Ambiente na Europa-Situação e Perspectivas 2010: Síntese. Agência Europeia do Ambiente, Copenhaga, 2010.

EUROPEAN COMMISSION. EU focus on waste management. Luxembourg: Office for Official Publications of the European Communities. p. 18, ISBN 92-828-4825-6, 1999.

EUROPEAN COMMISSION. The European Climate Change Programme. Brussels: European Commission, 2006.

EZEBILO, E. E., ANIMASAUN, E. D. Households' perceptions of private sector municipal solid waste management services: a binary choice analysis. International Journal of Environment Science and Technology, v.8, p.677-686, 2011.

FERRER, G., AYRES, R. U. The impact of remanufacturing in the economy. Ecological Economics, v.32, Issue 3, p. 413-429, ISSN 0921-8009, 2000.

FISCHER, C. Emissions pricing, spillovers, and public investment in environmentally friendly technologies. Energy Economics, v.30, n.2, p. 487-502, ISSN 0140-9883, 2008.

FISCHER-Kowalski M, AMANN, C. Beyond IPAT and Kuznets curves: globalization as a vital factor in analysing the environmental impact of socio-economic metabolism. Population and Environment, 23, p.7-47, 2001.

FOLZ, D. Municipal recycling performance: a public sector environmental successes story. Public Administration Review. 59, p. 336-345, 1999. 
GANDY, M. Recycling and the politics of urban waste. Earthscan Publications, London. 1994.

GARECHANA, G., RIO-BELVER, R., CILLERUELO, E., GAVILANES-TRAPOTE, G. Capturing waste recycling science. Technological Forecasting and Social Change, v.81, p. 250-258, ISSN 0040-1625, 2014.

GUNNINGHAM, N., GRABOSKY, P. Smart Regulation: Designing Environmental Policy. Oxford: Clarendon Press / Oxford University Press, 1998.

HANSEN, W., CHRISTOPHER, M., VERBUECHELN, M. EU Waste Policy and Challenges for Regional and Local Authorities Ecologic-Centre for International and European. Environmental Research, Berlin, 2002.

HASS, D., MURPHY, F., LANCIONI, R. Managing reverse logistic channels with data envelopment analysis. Transportation Journal. 42 (3), p. 105-113, 2003.

HERVANI, A. A. Can oligopsony power be measured? The case of U.S. old newspapers market. Resources, Conservation and Recycling, 44 (4), p. 343-380, 2005.

HIGHFILL, J., MCASEY, M. Municipal waste management: recycling and landfill space constraints. Journal of Urban Economics. v.41, n.1, p. 118-136, 1997.

JAFFE, A. B., NEWELL, R. G., STAVINS, R.N. A tale of two market failures: Technology and environmental policy. Ecological Economics, V. 54, Issues 2-3, p. 164-174, ISSN 0921-8009, 2005.

JENKINS, R., MARTINEZ, S., PALMER, K., PODOLSKY, M. The determinants of household recycling: A materialspecific analysis of recycling program features and unit pricing. Journal of Environmental Economics and Management 45 (3) p. 294-318, 2003.

KAIKA, D., ZERVAS, E. The Environmental Kuznets Curve (EKC) theory-Part A: Concept, causes and the CO2 emissions case. Energy Policy, V. 62, p. 1392-1402, ISSN 0301-4215, 2013.

LAVEE, D. Is municipal solid waste recucling ecomically efficient?” Environmental Management, 40, p. 926-943, 2007.

LAVEE, D., KHATIB, M., Benchmarking in municipal solid waste recycling. Waste Management, 30 (11), p. $2204-$ 2208, 2010.

LEHMANN, P. Justifying a policy mix for pollution control: a review of economic literature. Journal of Economic Surveys, 26: 71-97, 2012.

LINO, F. A. M., ISMAIL, K. A. R. Energy and environmental potential of solid waste in Brazil. Energy Policy, 39 (6), 3496-3502, 2011.

LEIGH, R. Economic growth as environmental policy? Reconsidering the Environmental Kuznets Curve. Journal of Public Policy, 24, p. 27-48, 2004.

MARQUES, R. C., CRUZ, N. F., Carvalho, P. Assessing and exploring (in)efficiency in Portuguese recycling systems using non-parametric methods. Resources, Conservation and Recycling, v.67, p. 34-43, ISSN 0921-3449, 2012.

MASSARUTTO, A. Waste management as a public utility: options for competition in an environmentally-regulated industry. Utilities Policy, 15, p. 9-19, 2007.

MASSARUTTO, A., DE CARLI, A., GRAFFI, M. () "Material and energy recovery in integrated waste management systems: A life-cycle costing approach. Waste Management, V. 31, Issues 9-10, p. 2102-2111, ISSN 0956-053X, 2011.

MASUI, T., MORITA, T., KYOGOKU, J. Analysis of recycling activities using multi-sectoral economic model with material flow. European Journal of Operational Research, V. 122, Issue 2, p. 405-415, ISSN 0377-2217, 2000.

MAZZANTI, M., ZOBOLI, R. Delinking and environmental Kuznets curves for waste indicators in Europe. Sciences Environmental, v. 2, n4, 2005.

MAZZANTI, M., ZOBOLI, R. Waste generation, waste disposal and policy effectiveness: evidence on decoupling from the European Union. Resources, Conservation and Recycling. v.52, n.10, p. 1221-1234, 2008.

MAZZANTI, M., MONTINI, A., ZOBOLI, R. Municipal waste generation and socioeconomic drivers: Evidence from comparing Northern and Southern Italy. Journal of Environment and Development, 17 (1), p. 51-69, 2008a

METIN, E., EROZTURK, A., NEYIM, C. Solid waste management practices and review of recovery and recycling operations in Turkey. Waste Management, v.23, n.5, p. 425-532, 2003

NAKAMURA, S. An interindustry approach to analyzing economic and environmental effects of the recycling of 
waste. Ecological Economics, v.28, n.1, p. 133-145, ISSN 0921-8009, 1999

NESTOR, D. Partial static equilibrium model of newsprint recycling. Applied Economics, v.24, n.4, p. 411-417, 1992

OKUDA, I., THOMSON, V. E. Regionalization of Municipal Solid Waste Management in Japan: Balancing the Proximity Principle with Economic Efficiency. Environmental Management. 40, p. 12-19, 2007.

PEARCE, D. W. Does European Union waste policy pass a cost benefit test?. World Economics, 15, p. 115-137, 2004.

PERETZ, J. H., TONN, B. E., FOLZ, D. H. (2005), "Explaining the performance of mature municipal solid waste recycling programs. Journal of Environmental Planning and Management, v.48, n.15, p. 627-650

PEROTTO, E., CANZIANI, R., MARCHESI, R., BUTELLI, P. Environmental performance, indicators and measurement uncertainty in EMS context: a case study. Journal of Cleaner Production, 16 (4), p. 517-530, 2008.

PIER, W.J., VERNON, R.B., WICKS, J.H. An empirical Comparison of Government and Private Production Efficiency. National Tax Journal, v.27, n.4, p. 653-656, 1974.

PLOURDE, C. G. A model of waste accumulation and disposal. Canadian Journal of Economics, v.5, n.1, p. 119$125,1972$.

READ, A. D., PHILLIPS, P., ROBINSON, G. Landfill as a future waste management option in England: the view of landfill operators. Resources, Conservation and Recycling, v.20, n.3, p. 183-205, ISSN 0921-3449, 1997.

SEPPÄLÄ, T., HAUKIOJA, T., KAIVO-OJA, J. The EKC hypothesis does not hold for direct material flows: environmental Kuznets curve hypothesis test for direct material flows in five industrial countries. Population and Environment, v.23, n.2, p. 217-238, 2001.

SHMELEV, S.E., POWELL, J.R. Ecological-economic modelling for strategic regional waste management systems. Ecological Economics. v.59, p. 115-130, ISSN 0921-8009, 2006.

SIMÕES, P., DE WITTE, K., MARQUES, R.C. Regulatory structures and operational environment in the Portuguese waste sector. Waste Management, v. 30, n.6, p. 1130-1137, ISSN 0956-053X, 2010.

SIMÕES, P., MARQUES, R. C. On the economic performance of the waste sector. A literature review. Journal of Environmental Management, v.106, p.40-47, 2012.

SMITH, V.L. Dynamics of waste accumulation: disposal versus recycling. Quarterly Journal of Economics, v.86,n.4, p. 600-616, 1972.

STEVENS, B.J. Scale, market structure, and the cost of refuse collection. Review of Economics and Statistics, v.60, n.3, p. 438-448, 1978.

SZYMANSKI, S., WILKINS, S. Cheap rubbish? Competitive tendering and contracting Out in Refuse Collection1981-88. Fiscal Studies, v.14, n.3, p.109-130, 1993.

TICKNER, G., MCDAVID, J. Effects of scale and market structure on the costs of residential solid waste collection in Canadian cities. Public Finance Quarterly, v.14, p. 371-393, 1986.

VICKERS, J., YARROW, G. Economic Perspectives on Privatization. Journal of Economic Perspectives. v.5, n.2, 1991.

WALLS, M. How local governments structure contracts with private firms: economic theory and evidence on solid waste and recycling contracts. Public Works Management. v.9, p. 206-222, 2005.

WAlls, M., MACAUley, M., ANDERSON, S. Private Markets, Contracts, and Government Provision: What Explains the Organization of Local Waste and Recycling Markets?. Urban Affairs Review, v.40, n.5, p.590-613, 2005.

WANG, P., BOHARA, A., BERRENS, R., GAWANDE, K., A risk based environmental Kuznets curve for US hazardous waste sites". Applied Economics letters, v.5, n.12, pp. 761-763, 1998.

WENG, Y.C., FUJIWARA, T. Examining the effectiveness of municipal solid waste management systems: an integrated cost-benefit analysis perspective with a financial cost modeling in Taiwan. Waste management, v.31, pp. 1393-1406, 2011.

YANRONG, W., CUILI, W., HAN, W. Research on the Quantitative Relationship between the Generation of Industrial Solid Waste and Per Capita GDP of Henan Province. Energy Procedia, Vol. 5, pp. 593-597, ISSN 1876-6102, 2011. 